NOTAS

\title{
Antecedentes sobre la recolección y comercialización de productos forestales no madereros (PFNM), en localidades rurales de la región del Maule, Chile central
}

\author{
Background on the collection and marketing of non-timber forest products (NTFPs) \\ in rural towns in the Maule Region, Central Chile
}

\author{
Marisol Muñoz *a, Darío Aedo a, José San Martín ${ }^{\text {b }}$

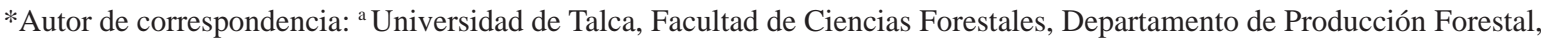 \\ Avenida Lircay s/n, Talca, Chile, tel.: 56712200 368, mmunoz@utalca.cl \\ b Universidad de Talca, Instituto de Biología Vegetal y Biotecnología, Talca, Chile.
}

\begin{abstract}
SUMMARY
The importance of non-timber forest products (NTFPs) for the welfare of rural communities is globally recognized. The aim of this study was to provide a background of the collection and marketing of NTFPs in rural towns in the Maule Region. A representative sample of $0.2 \%$ of the regional rural population was surveyed. The results revealed that NTFP collectors were $89 \%$, with large participation of women. Collected and sold NTFPs come from the introduced species Rubus ulmifolius and Rosa rubiginosa; and from the native Cyttaria espinosae, Gevuina avellana and Peumus boldus. Among provinces, people showed different preferences in terms of harvested and sold species. The results revealed the important value of NTFPs for rural population.
\end{abstract}

Key words: resource conservation, sustainable use, NTPFs.

\section{RESUMEN}

La importancia de los productos forestales no madereros (PFNM) para el bienestar de las comunidades rurales se reconoce a nivel mundial. El objetivo del presente estudio fue aportar antecedentes sobre la recolección y comercialización de PFNM preferidos, en poblados rurales de la región del Maule. Se levantó una muestra que representa el 0,2% de la población rural regional. Los resultados revelaron que un 89 \% eran recolectores, con gran participación de mujeres. Los PFNM recolectados y vendidos provienen de las especies introducidas Rubus ulmifolius y Rosa rubiginosa; y de las nativas Cyttaria espinosae, Gevuina avellana y Peumus boldus. Entre las provincias, el comportamiento de los pobladores se diferenció en términos de las especies más recolectadas y vendidas. Los resultados revelaron la importancia del valor de los PFNM para la población rural.

Palabras clave: conservación del recurso, uso sustentable, PFNM.

\section{INTRODUCCIÓN}

Los productos forestales no madereros (PFNM) se definen como "bienes de origen biológico, distintos de la madera, derivados del bosque, de otras áreas forestales y de los árboles fuera de los bosques. Estos productos pueden recolectarse en forma silvestre o producirse en plantaciones forestales o sistemas agroforestales" (FAO 1999). Su cosecha representa una importante fuente de ingresos para millones de personas en todo el mundo, tanto en ecosistemas áridos (Olujobi 2012, Adam et al. 2013) como tropicales (Figueroa et al. 2010, Ferraz et al. 2012). Los bosques, además de generar productos maderables y servicios ambientales, tienen gran valor social para las comunidades rurales que realizan la recolección, proporcionando fuentes de trabajo a ancianos, mujeres y niños (Campos 1998) y contribuyendo significativamente al estilo de vida de la población rural (Olujobi 2012), en la mitigación de la pobreza (Adam et al. 2013), y con gran potencial para un desarrollo rural sustentable (Stryamets 2012).

Desde tiempos ancestrales el hombre ha desarrollado actividades de recolección para su subsistencia y para el comercio (Ticktin 2004, Ortiz et al. 2008) y Chile no es la excepción. El historiador Rosales, en el año 1877, describe los usos madereros y no madereros, que los indígenas daban a distintas especies del bosque nativo, como alerce (Fitzroya cupressoides (Mol.) I.M. Johnston), ciprés de la cordillera (Austrocedrus chilensis (D. Don) Pic.Serm. et Bizzarri), palma chilena (Jubaea chilensis (Mol.) Baill.), 
maqui (Aristotelia chilensis (Mol.) Stuntz) y murtilla (Ugni molinae Turcz.), entre otras (Rosales 1877).

En la región del Maule existen 384.714 ha de bosque nativo, con desigual distribución entre las provincias. Este bosque es fuente de diversos PFNM, pero escasos estudios lo reportan. En las localidades de "El Peñasco" y "Llepo", de la comuna de Linares, Troncoso (2003) da cuenta que la totalidad de familias entrevistadas desarrollaban labores extractivas de frutos comestibles, hongos comestibles y plantas medicinales, situación que podría extrapolarse a otros sectores precordilleranos de la región. Para el territorio sur del Maule, conformado por las comunas Empedrado, Cauquenes, San Javier, Pelluhue y Chanco, el Instituto Forestal (2005) da cuenta sobre el potencial productivo apícola y hongos silvestres. El rubro más importante es la recolección de hongos silvestres, cuya comercialización local genera hasta un 50 \% de ingresos a la economía familiar. En la recolección participan mayoritariamente mujeres. Los hongos son colectados en plantaciones de Pinus radiata D. Don, como las especies Suillus luteus (L. ex Fr.) Gray, Boletus granulatus (L. ex Fr.) Kuntze, Lactarius deliciosus (L. ex Fr.) Gray y en el bosque nativo Morchella conica Pers., Cyttaria espinosae Lloyd, Clavaria sp. Fr. y Gyromotra antarctica Rehm. En relación con la actividad apícola se estableció que en las cinco comunas el principal producto obtenido es la miel, en desmedro de otros como jalea real, polen, propóleo y servicios de polinización.

Ante la escasez de información sobre la diversidad de PFNM y su persistencia para la región del Maule, el objetivo de este trabajo es determinar la variedad de productos, tanto nativos como introducidos, cosechados por la población rural vecina a bosques naturales, así como establecer probables diferencias en la recolección y comercialización entre las provincias de Talca, Cauquenes, Curicó y Linares. La hipótesis planteada es que las diferencias entre los bosques de cada provincia influyen en el aprovechamiento de PFNM que realizan las poblaciones locales.

\section{MÉTODOS}

La región del Maule se ubica entre los $34^{\circ} 41^{\prime}$ y $36^{\circ} 33^{\prime}$ de latitud Sur, y entre los $70^{\circ} 20^{\prime}$ y los $72^{\circ} 43^{\prime}$ de longitud Oeste, con una superficie de $30.296 \mathrm{~km}^{2}$ que representa el 4 \% del país. Tiene una población de 908.097 habitantes, con la mayor población rural del país (305.077 habitantes) (INE 2002). Para efectos administrativos, la región está dividida en cuatro provincias y 30 comunas.

De oeste a este, el relieve de la región presenta planicies costeras, Cordillera de la Costa, Depresión Intermedia, precordillera andina y Cordillera de Los Andes, generando diversidad de condiciones ambientales y ecosistemas.

En la composición del bosque nativo maulino convergen especies típicas de la zona mediterránea central con otras más características del sur del país, por lo que se presenta una vegetación de transición. En el bosque dominan especies del género Nothofagus. Por otro lado, es posible en- contrar otras formaciones de carácter caducifolio o siempreverde y comunidades con especies esclerófilas (San Martín y Donoso 1996).

Previo al muestreo, se visitaron todas las comunas, para rescatar información necesaria para la selección de las localidades. En estas visitas se validó el cuestionario diseñado para el levantamiento de la información. Con base en lo anterior, se seleccionaron 45 localidades, con población rural y bosque nativo en un radio de 5 a $10 \mathrm{~km}$, en estos lugares se encuestó a los lugareños, usando un cuestionario semiestructurado, sobre qué recolectan, quiénes les ayudan, destino de lo recolectado, problemas en la recolección y venta. En forma introductoria a la encuesta, se informó a los pobladores que el interés se centraba sobre la cosecha de PFNM en el bosque nativo, por lo que fue necesario explicarles la diferencia con las plantaciones.

Los resultados se basan en la información voluntaria, proporcionada por 501 pobladores rurales, mayores de 18 años, que asistieron a las reuniones y aceptaron participar en este trabajo. El análisis de los datos se realizó a base de estadística descriptiva para confeccionar tablas de frecuencias y porcentajes. Se realizaron análisis de asociación para las respuestas de los encuestados. Así, utilizando los estadísticos chi-cuadrado, obtenidos de tablas de contingencias, se agruparon las respuestas por provincias y se determinaron similitudes de comportamiento.

\section{RESULTADOS}

Un alto porcentaje de los encuestados (89 \%) señaló realizar actividad de recolección, siempre o a veces. Se encontraron diferencias entre provincias $(P=0,0012)$; la mayoría de los pobladores pertenecientes a Linares recolectan productos del bosque (93\%) y los que menos lo hacen son de las comunas de Curicó (77 \%). Para Cauquenes y Talca se observó un comportamiento similar $(P=0,8430)$, con un 90 a $92 \%$ de población recolectora. También se encontraron diferencias por sexo $(P<0,001)$, las mujeres recolectoras superaron a los hombres (91\% vs $83 \%)$. Los encuestados señalaron que la recolección se realiza, de preferencia, como una actividad familiar (68 \%) y, en menor proporción en forma solitaria (30 \%) o en compañía de vecinos o medieros (2\%).

$\mathrm{Al}$ ordenar, de mayor a menor, el número de citaciones de las especies frecuentemente recolectadas por los pobladores se originó el siguiente ranking (cuadro 1): Rubus ulmifolius Schott (fruto comestible), Cyttaria espinosae (hongo comestible), Gevuina avellana Mol. (fruto comestible, hojas ornamentales), Rosa rubiginosa L. (fruto comestible), Peumus boldus (fruto comestible, hojas para uso medicinal), Aristotelia chilensis (fruto comestible), Ugni molinae (fruto comestible), Ramaria flava (Schff. ex Fr.) Quél. (hongo comestible), Lardizabala biternata Ruiz et Pav. (fruto comestible) y Cryptocarya alba (Mol.) Looser. (fruto comestible, hojas para uso medicinal). Se encontraron diferencias entre las provincias $(P<0,001)$. 
Los encuestados asociaron principalmente sus problemas de recolección con la accesibilidad al bosque (46 \%), señalando la lejanía, caminos inadecuados y polvorientos y falta de permisos para acceder a los predios. Como dificultades para la recolección misma (27 \%) se mencionan escasez de los productos o especies, condiciones climáticas adversas, ataques de Vespula germanica F. (avispa chaqueta amarilla), corto periodo de recolección y sobrepoblación de Apis mellifera L. Por otro lado, también declararon falta de interés en recolectar (20\%), debido a problemas para la comercialización de los productos obtenidos y, en último término, problemas de los mismos recolectores (7\%), como enfermedades, escasez de mano de obra y falta de herramientas. La provincia de Talca presentó un comportamiento diferente al resto $(P=0,4587)$, los lugareños atribuyen principalmente sus problemas al proceso de recolección y a la accesibilidad, en las provincias restantes destacan las limitaciones de accesibilidad al bosque.

Sobre el destino de la recolección, no se encontraron diferencias entre provincias $(P=0,4446)$. Se declaró venta y autoconsumo en un $43 \%$, un $33 \%$ expresó que sólo se autoabastece y un $24 \%$ manifestó que sólo vende lo recolectado. Los encuestados reconocieron la venta de 25 PFNM, algunos con valor agregado, aunque con escasa representatividad, como mermeladas $(4,4 \%)$, artesanías $(0,2 \%)$, cestería $(0,5 \%)$, harina de avellana $(0,2 \%)$. También se mencionó la venta de ramas de avellano (2\%), gusanos de tebo (Chilecomadia moorei (Silva)) (0,7\%), tierra de hojas (0,5\%), plántulas extraídas del bosque (0,2 \%), entre otros. Los productos más relevantes son los frutos de Rubus ulmifolius, Rosa rubiginosa, Gevuina avellana y el hongo Cyttaria espinosae (cuadro 2). Se encontraron

Cuadro 1. Especies citadas por los entrevistados. Número de citaciones por provincia de las especies frecuentemente recolectadas. Species cited by respondents. Number of citations per province of frequently collected species.

\begin{tabular}{llccccc}
\hline \multirow{2}{*}{ Especie } & Nombre común & \multicolumn{2}{c}{ Número de citaciones por provincia } & & \\
\cline { 3 - 7 } & & Cauquenes & Curicó & Linares & Talca & Total \\
\hline Rubus ulmifolius & Mora & 18 & 45 & 63 & 68 & 194 \\
Cyttaria espinosae & Dihueñe & 52 & 5 & 46 & 31 & 134 \\
Gevuina avellana & Avellana & 49 & 11 & 17 & 48 & 125 \\
Rosa rubiginosa & Mosqueta & 30 & 10 & 41 & 36 & 117 \\
Peumus boldus & Boldo & 4 & 5 & 43 & 6 & 58 \\
Aristotelia chilensis & Maqui & 22 & 6 & 10 & 16 & 54 \\
Ugni molinae & Murtilla & 17 & 3 & 23 & 3 & 46 \\
Ramaria flava & Changle & 18 & 0 & 22 & 6 & 46 \\
Lardizabala biternata & Cóguil & 0 & 7 & 2 & 8 & 17 \\
Cryptocarya alba & Peumo & 0 & 4 & 0 & 13 & 17 \\
Otras & - & 15 & 22 & 44 & 23 & 104 \\
\hline Total & & 225 & 118 & 311 & 258 & 912 \\
\hline
\end{tabular}

Cuadro 2. Productos forestales no madereros (PFNM) citados por los entrevistados. Número de citaciones por provincia de los principales PFNM comercializados.

Species cited by respondents. Number of citations per province of frequently collected species.

\begin{tabular}{|c|c|c|c|c|c|c|}
\hline \multirow{2}{*}{ PFNM } & \multirow{2}{*}{ Nombre común } & \multicolumn{4}{|c|}{ Número de citaciones por provincia } & \multirow[b]{2}{*}{ Total } \\
\hline & & Cauquenes & Curicó & Linares & Talca & \\
\hline Rubus ulmifolius & Mora & 3 & 32 & 65 & 51 & 151 \\
\hline Rosa rubiginosa & Mosqueta & 16 & 0 & 17 & 13 & 46 \\
\hline Gevuina avellana & Avellana & 24 & 1 & 11 & 5 & 41 \\
\hline Cyttaria espinosae & Dihueñe & 21 & 0 & 0 & 14 & 35 \\
\hline Otros & - & 18 & 19 & 23 & 79 & 139 \\
\hline Total & - & 82 & 52 & 116 & 162 & 412 \\
\hline
\end{tabular}


diferencias entre las cantidades de productos citados por provincias $(P<0,001)$.

Todos los encuestados que realizan venta de productos reconocen como problema mayor los bajos precios. Así lo señalan los pobladores de Curicó y Talca $(P=0,3237)$; en cambio, en Cauquenes y Linares $(P=0,4669)$, agregaron la accesibilidad (traslado, lejanía, mal estado de caminos), exigencias en calidad y aspectos sanitarios y falta de compradores en las localidades.

\section{DISCUSIÓN}

El alto porcentaje de recolectores (89 \%) valida la importancia de los PFNM para los pobladores rurales de la región. La recolección, para autoconsumo o comercialización, muestra gran significancia social, participan distintas generaciones, no se requiere mayor calificación, generalmente se transmite de padres a hijos, siendo una actividad que une a la familia rural. En Chile, esta actividad genera empleo e ingresos a más de 200 mil habitantes rurales (Valdebenito 2013).

Las especies más recolectadas y vendidas son $R$. rubiginosa y R. ulmifolius, ambas introducidas e invasoras del bosque nativo, que también se encuentran en áreas silvestres protegidas (Jiménez et al. 2013). La primera, puede estar entremezclada con flora nativa, crece agresivamente y forma densos matorrales; y R. ulmifolius invade grandes áreas en poco tiempo, es difícil de erradicar e impide el crecimiento de especies nativas (Quiroz et al. 2009). Estas especies se mencionaron en localidades de las comunas costeras (Vichuquén, Constitución, Pelluhue, Cauquenes), del centro (San Javier, Río Claro) y precordillera andina (San Clemente, Colbún, Linares, Longaví).

Las diferencias de recolección entre provincias pueden explicarse, en parte, por el tipo de bosque que se encuentra en cada lugar y por las posibilidades de comercialización. A pesar de la diversidad florística del bosque maulino (San Martín y Donoso 1996, Stoll et al. 2006), fuente de múltiples productos y con variados usos, como medicinales, alimentarios, tintóreos, etc., se recolectan los de mayor demanda comercial, aunque los precios sean bajos según la visión de los entrevistados, ya que es una oportunidad de obtener ingresos. Para mejorar los precios de venta es necesario agregar valor a los productos (Valdebenito 2013), eliminar intermediarios, mejorar la asociatividad (Troncoso et al. 2010), aspectos que deberían abordarse en programas de extensión.

En la medida que el bosque brinde PFNM, que los lugareños los puedan aprovechar sustentablemente y les permitan obtener ingresos, favoreciendo la economía rural, se contribuye a una valorización positiva y de conservación del bosque. Pero, el reconocimiento sólo de la valoración económica puede incentivar la explotación no sustentable, afectando procesos ecológicos, por lo que es importante fomentar la valoración de los servicios ambientales y las buenas prácticas de extracción. A modo de ejemplo se puede mencionar que, en conversaciones sostenidas con los campesinos, ellos reconocen que después de un incendio se fomenta la aparición de Morchella conica Pers., hongo que tiene uno de los mejores precios del mercado, por lo tanto es necesario educar insistiendo en la sustentabilidad del recurso para evitar posibles quemas intencionadas. También se pudo constatar que los pobladores extraen tierra de hojas, helechos y plántulas de regeneración, sin fiscalización alguna, porque en la actualidad no existe en el país un marco normativo que regule la extracción y comercio de los PFNM, por lo que urge su implementación. Para ello, se necesitan estudios sobre la ecología y niveles sostenibles de cosecha de estos productos, en especial de las especies endémicas Peumus boldus, Lardizabala biternata y Cryptocarya alba; ya que una explotación desmedida puede ocasionar la desaparición de las especies del ecosistema (Ferraz et al 2012).

Los entrevistados reconocen como una fortaleza la existencia de bosque nativo en sus sectores, señalaron que les brindaba oportunidades para el turismo, belleza escénica y extracción de PFNM. Para que ello siga ocurriendo se requiere de un fuerte y decidido apoyo gubernamental para establecer programas de extensión permanentes en las comunidades rurales, que permitan educar sobre el manejo racional del bosque, fomentar el desarrollo de competencias y capacidades de los pobladores, fortalecer sus organizaciones sociales, incentivar emprendimientos locales que puedan generar empleos y mejorar la calidad de vida de los habitantes rurales de la región del Maule.

\section{AGRADECIMIENTOS}

Los autores agradecen el financiamiento otorgado al Proyecto 002-2011, por el Fondo de Investigación del Bosque Nativo y a los pobladores maulinos que participaron en el estudio.

\section{REFERENCIAS}

Adam YO, J Pretzsch, D Pettenella. 2013. Contribution of Non-Timber Forest Products livelihood strategies to rural development in drylands of Sudan: Potentials and failures. Agricultural Systems 117: 90-97.

Campos J. 1998. Productos Forestales No Madereros en Chile. Serie Forestal $N^{\circ} 10$. Santiago, Chile. Organización Mundial para la Alimentación y la Agricultura (FAO). 65 p.

CIREN (Centro de Información de Recursos Naturales, CL). 1990. Atlas agroclimático de Chile, Regiones IV a IX. Publicación N 87. Santiago, Chile. CIREN. 66 p.

FAO (Food and Agriculture Organization of the United Nations, IT). 1999. Hacia una definición uniforme de los productos forestales no madereros. Unasylva 198: 63-64.

Ferraz J, R Ferreira, M dos Santos, I Meunier. 2012. Usos de especies leñosas de la caatinga del municipio de Floresta en Pernambuco, Brasil: conocimiento de los indios de la aldea Travessão do Ouro. Bosque 33(2): 183-190.

Figueroa J, E Sanoja, L Delgado. 2010. Árboles utilizados 
como productos forestales no maderables en la cuenca alta del río Botanamo, Estado Bolívar, Venezuela. Acta Botánica Venezuelica 33 (1): 119-135.

INE (Instituto Nacional de Estadísticas, CL). 2002. Población y sociedad. Aspectos demográficos. Consultado 28 oct. 2013. Disponible en http://www.ine.cl/canales/chile_estadistico/ familias/demograficas vitales.php

INFOR (Instituto Forestal, CL). 2005. Potencial productivo de pequeños y medianos propietarios madereros y no madereros en la Región del Maule. Líneas base de los rubros maderero, apícola y hongos silvestres en el Territorio Maule Sur. Santiago, Chile. INFOR. 153 p.

Jiménez A, A Pauchard, A Marticorena, R Bustamante. 2013. Patrones de distribución de plantas introducidas en áreas silvestres protegidas y sus áreas adyacentes del centro-sur de Chile. Gayana Botanica 70(1): 110-120.

Olujobi OJ. 2012. Harvesting practices, utilization and conservation of NTFPs in Ekiti State, Nigeria. International Journal of Academic Research 4(1): 134-140.

Ortiz K, B Román, M Nahuelhual. 2008. Generación de ingreso rural a partir de la recolección de follaje ornamental de cuatro especies nativas de los bosques costeros del sur de Chile. Agro Sur 36(3): 168-177.

Quiroz CL, A Pauchard, A Marticorena et LA Cavieres. 2009. Manual de plantas invasoras del centro-sur de Chile. Concepción, Chile. Laboratorio de Invasiones Biológicas, Universidad de Concepción. 45 p.
Rosales D. 1877. Historia general de el Reyno de Chile: Flandes indiano. Valparaíso, Chile. Impr. del Mercurio. p: 221-250.

San Martín J, C Donoso. 1996. Estructura florística e impacto antrópico en el Bosque Maulino de Chile. In Armesto JJ, C. Villagrán, MTK Arroyo eds. Ecología de los bosques nativos de Chile. Santiago, Chile. Universitaria. p. 153-168.

Stoll A, C Sepúlveda, J San Martín. 2006. Patrón florísticoestructural de la vegetación nativa remanente en el límite norte del Bosque Templado Costero de Chile: el caso de la quebrada Cayurranquil (VII Región, Chile). Bosque 27(1): 64-71.

Stryamets N. 2012. Non-wood forest products for livelihoods. Bosque 33(3): 329-332.

Troncoso M. 2003. Selección de productos forestales no madereros con potencial económico-productivo en las localidades de "El Peñasco y Llepo" comuna de Linares, VII Región. Tesis Ingeniero Forestal. Talca, Chile. Facultad de Ciencias Forestales, Universidad de Talca. 87 p.

Troncoso L, M Hernández, F Milla. 2010. Cadena de valor de los productos no madereros del bosque nativo (PNMBN) para las familias campesinas de Alto Bío-Bío, Chile. Gestión Ambiental 20: 27-48.

Valdebenito G. 2013. Existencia, uso y valor de los productos forestales no madereros (PFNM) del bosque nativo en Chile. Consultado 29 oct. 2013. Disponible en http://www.gestionforestal.cl:81/pfnm/otros/seminarios_publicaciones/ publicaci\%C3\%B3n\%20IUFRO\%20PFNM\%20Chile.pdf 
